\title{
Chapter
}

12

\section{Systemic and Socially Aware Perspective for Information Systems}

\author{
Roberto Pereira, M. Cecília C. Baranauskas
}

\begin{abstract}
Information and Communication Technology triggers changes affecting the environment in which it operates and the people who live in this environment - even those who do not use it. Information Systems are available worldwide, shaping organizations, business, governments and societies. Therefore, designing and using information systems has assumed new dimensions in terms of complexity and has required a wider and deeper understanding of the ethical and social responsibilities of those who design them. In this Grand Challenge, we argue that technical, social, legal and ethical problems in the development and use of information systems arise from a narrow understanding of information systems that does not favor the social world in which they exist and are used. Instead, we must develop and adopt a systemic view for Information Systems that considers a technical solution as part of a more complex social system in which people live and interact; a socially aware view able to understand and anticipate the demands, challenges, problems and opportunities of a society increasingly connected and mediated by interactive technologies.
\end{abstract}

\subsection{Introduction}

“(...) science and technology is not given. It was made by people like us. If it's not doing for us what we want, we have a right and a responsibility to change it." (Mike Cooley ${ }^{1}$. December 9th, 1981)

The call for papers for the Grand Challenges in Information Systems in Brazil (GranDSI-BR) highlights that "Information Systems (IS) have been one of the main agents of economic growth and social transformations in Brazil and the world in recent decades, and the perspective is clear that they will remain so in the next years". In recent years, the changes computing systems have triggered in economic, ethical, political issues, as well as in organized societies, have become more evident and critical [Pereira et al. 2015]. IS have caused profound changes in the way

\footnotetext{
${ }^{1}$ http://www.rightlivelihoodaward.org/laureates/mike-cooley/ Last access on 02 February 2017.
} 
organizations function, businesses are defined, carried out, and regulated, and life in society is organized. Although IS is both a well-established and recognized discipline and area of computing technology, it is constantly changing, requiring permanent (re)positioning to remain useful and responsive to the demands of an increasingly technology-mediated society.

In 2015, the Brazilian government launched the eSocial ${ }^{\circ}$ platform² ${ }^{2}$, designed to manage and facilitate the payment of taxes for domestic workers. Imposed by the federal government as a substitute for existing methods (e.g., manual payments, automatic debit), the system was launched in November 2015 and, due to technical problems, its poor user interface, a lack of understanding of the formal and informal aspects of the problem domain etc., about $1 / 3$ of its registered employers were not able to pay their taxes and late payments were subject to fines. An article published in "O Globo" newspaper (in Portuguese) highlighted "gross errors" as the result of a poor system design, such as not considering particularities of the Brazilian zip code address, ignoring different e-mail address format, and not even paying attention to the level of knowledge and experience in the use of computer systems by the potential users, namely, the diversity of the Brazilian population. In this case, people were forced to use a system that did not allow them to comply with the imposed norms. In practice, people were fined for being prevented from complying with their obligations, not to mention the emotional and social impact on the ones who used to pay their taxes at a bank branch, and do not have access to computer technology (e.g., elderly people). The system which was originally designed to make tax payers' lives easier and to favor formal registration of the domestic work class, became an example of how an interactive computing system can be imposed and create barriers and complicate a task people had few problems performing in the past.

In September 2015, Volkswagen ${ }^{\circ}$, one of the largest companies in the automotive industry, admitted that it had developed a software system to manipulate pollutant emissions rates for some of its vehicles ${ }^{3}$. Installed in 11 million diesel vehicles around the world, the software identifies when vehicles are being tested, manipulating pollutant emission data in order to meet the standards and laws required in specific countries, such as the USA. However, when the car is in its normal usage mode, i.e., on the streets and highways, the device is turned off and gas emissions may be 10 to 40 times greater than those identified in laboratory tests. This case raised concerns and discussions on the critical importance of considering ethical issues and responsibilities in Information Communication Technology (ICT) design, an example of how a software system can (be designed to) cheat technical and legal norms.

In March 2016, Microsoft ${ }^{\circ}$ had to deactivate Tay (acronym of "thinking about you"), its chatbot, as it became racist, cited Hitler and started supporting Donald Trump's immigration plans a few hours after it had been launched online. The chatbot was targeted at 18 to 24 -yearold people in the US as part of research on conversational understanding, designed to engage in conversations via twitter and to learn from these interactions. According to Microsoft ${ }^{4}$, it planned and implemented several filtering features and conducted extensive user studies with diverse user groups when designing Tay. However, Twitter ${ }^{\bullet}$ users were able to explore

\footnotetext{
${ }^{2}$ http://www.esocial.gov.br/

${ }^{3}$ http://www.bbc.com/news/business-34324772

${ }^{4}$ http://blogs.microsoft.com/blog/2016/03/25/learning-tays-introduction/\#PmLozZ6PuFpz4ZY5.99
} 
vulnerabilities in Tay, "teaching" the chatbot in a way Microsoft was not able to anticipate. Microsoft took "full responsibility for not seeing this possibility ahead of time", claiming that Artificial Intelligent systems feed off of both positive and negative interactions with people, making the challenges as much social as technical. Microsoft admitted: "Tay is now offline, and we'll look to bring Tay back only when we are confident we can better anticipate malicious intent that conflicts with our principles and values". However, Microsoft alerts: "we will do everything possible to limit technical exploits but also know we cannot fully predict all possible human interactive misuses without learning from mistakes".

Decades ago, Papanek (1971) drew attention to the field of industrial design, arguing that designers are not free of responsibility for the products they create and deliver to the world. Criticizing a culture centered on economic and technical issues, the author pointed out to the impacts caused by the design of products that promote bad and harmful behaviors, or even mutilate and kill people, neglecting the social context of design, the target audience and society in general. For Papanek, it was mandatory to start thinking about a socially and environmentally responsible design of products, tools and infrastructure, and to recognize designers' social and moral responsibility in this process.

The examples cited above indicate that we still live at a time analogous to that criticized by Papanek (1971), but on an ever-increasing scale. Technology triggers changes affecting the environment in which it is inserted and the people who live in this environment - even the ones who do not use it. Ubiquitous Computing, Wearable Computing, Social Applications, Ambient Assisted Living, and the Internet of Things are some examples of how computing information systems have permeated all aspects of personal and collective life in more complex and connected ways. The challenges and problems of an ICT-mediated society can neither be solved from a technically-centered perspective - ignoring the social world in which solutions are used and people live, nor be addressed with specific approaches in an isolated and fragmented way.

A technical-centered perspective has been a standard practice in the domain of Information Systems, as well as in Computer Science, partly due to a lack of knowledge and support tools to adopt (and practice) a systemic and socially aware perspective. Consequently, it is difficult to design solutions and obtain results that make sense to the different stakeholders interested in the problem domain and then produce the expected benefits for the social, technological and scientific development of a society.

Ethics, human values, and culture are intertwined with each other [Pereira and Baranauskas 2015], and seem to be critical issues for a socially aware understanding of Information Systems. Areas such as architecture, medicine, engineering and law are concerned about investigating how to build better physical spaces, provide better quality of life, developing more efficient technologies, and ensuring better organization and conditions for human life. Information Systems, as both a powerful tool and an area in itself, must take such issues seriously, encouraging its professionals - in their different roles - to be aware and concerned about how to use knowledge and solutions to improve the lives of people in their environments in a way that makes sense to them and not trigger adverse effects on the lives of individuals and communities.

In the field of Human-Computer Interaction ( $\mathrm{HCl}$ ), different initiatives have been made 
to identify and inspire directions in $\mathrm{HCl}$ for the near future. Bannon (2011), for instance, argues for a reformulation (reimagination) of the $\mathrm{HCl}$ discipline, exploring new forms of living with/through technologies. Bødker (2006) talks about a third wave in $\mathrm{HCl}$ in which new elements of human life are included, such as culture, emotion, and experience, which require new forms of understanding design of computing technology able to account for such elements. In 2007, researchers from academia and industry, from several countries and with different backgrounds, joined efforts to understand and idealize $\mathrm{HCl}$ in 2020 (2009); and in 2012, the Brazilian $\mathrm{HCl}$ research community prospected grand research challenges for $\mathrm{HCl}$ in Brazil for the next 10 years [Baranauskas et al. 2015]. These initiatives suggest that considering human values and understanding the cultural context of design are challenges for the area itself and for all those involved with the design, evaluation and use of interactive computing technology. Such concerns are naturally extended to the IS domain.

Particularly in the field of IS, several initiatives have been made over the last four decades to discuss and inspire the development of this field. Becker et al. (2015) presented an overview of such initiatives and presented a study with 143 IS academics to prospect grand challenges for the IS field and discipline. The authors argued that the IS field is characterized by its social context, and identified 21 possible Grand Challenges they grouped into 4 main categories: i) socio-technical challenges, ii) IS infrastructure challenges, iii) societal and ecological challenges, and iv) social and affective challenges. For Becker et al. (2015), the results suggested that the old debate on the identity of IS had not yet been overcome, as results highlight a strong concern of IS with itself (i.e., its identity, relevance, foundational theory, methodological pluralism). These results suggest the identification of challenges, but not necessarily of Grand Challenges. Nevertheless, they highlight IS as a practical discipline that needs to serve the goals of society, requiring a deep, critical and socially responsible understanding of what society requires.

In fact, when discussing a future agenda for the IS field, Walsham (2012) questions whether we, as a community, "are making a better world with ICTs". The author highlights that dramatic changes have occurred over the last decades, with ICTs being spread over almost all countries and becoming pervasive in many areas of human activity. Such changes raised concerns that the research contribution of the field could have got lost and that the field itself could be in decline, and the author suggested that an agenda focused on ethical goals should be mandatory for the field to remain relevant and offer a unique contribution.

In this chapter, we highlight as a Grand Challenge the concept and adoption of a Systemic and Socially Aware Perspective for Information Systems, focusing on ethical goals and considering a technical solution as being part of a more complex social system in which people live and interact. Such a perspective could serve not only as a theoretical and methodological framework, but also as goal to pursue, helping to characterize the area's unique contribution to science and technology for a better world.

\subsection{Background}

In 1959, Hall had already found that introducing or changing a technology is the most powerful way to promote changes in a culture and to redefine a society. In different ways, contexts, and dimensions, technology has affected our lives: the ways we work, study, eat, interact with each 
other, understand time and space, and live. More and more life is being mediated and influenced by ICTs.

When studying and discussing culture as a form of nonverbal communication, Hall (1959) introduced the notions of informal, formal, and technical levels in which humans operate and understand the world. The informal represents the culture, values, habits, beliefs, behavioral patterns of people and other aspects that are usually difficult to describe and even identify. The formal represents aspects that are well established and accepted, becoming social conventions, norms, or laws. The technical level represents aspects that are so formalized that they can be automated or approached in a technical way. According to Hall, each level is present in any situation, but one always dominates at a given instant of time and we deal with them separately - i.e., when we focus our attention on one level, the other two levels are in the background, intertwined with the one in focus. The author explains that, sometimes, the shifts (and boundaries) between these levels are subtle and rapid, but such shifts are the basic requirement to understand the process of change in a society.

Organizational Semiotics theory proposes a structure named Organizational Onion [Stamper, 2000], also called "Semiotic Onion", to explain how the informal, formal and technical levels coexist in the context of organizations and information systems - see Figure 12.1. The central idea is that any technical artifact is embedded in a formal system that, in turn, is embedded in an informal one. It means that a technical information system, for example, is capable of automating or implementing only part of a formal information system that exists in society and is composed of norms, rules, laws and regular patterns of behavior, which in turn, exist in an informal information system that is part of the social world where people live, interact with each other, develop their values, have their needs, expectations, dreams, intentions, etc.

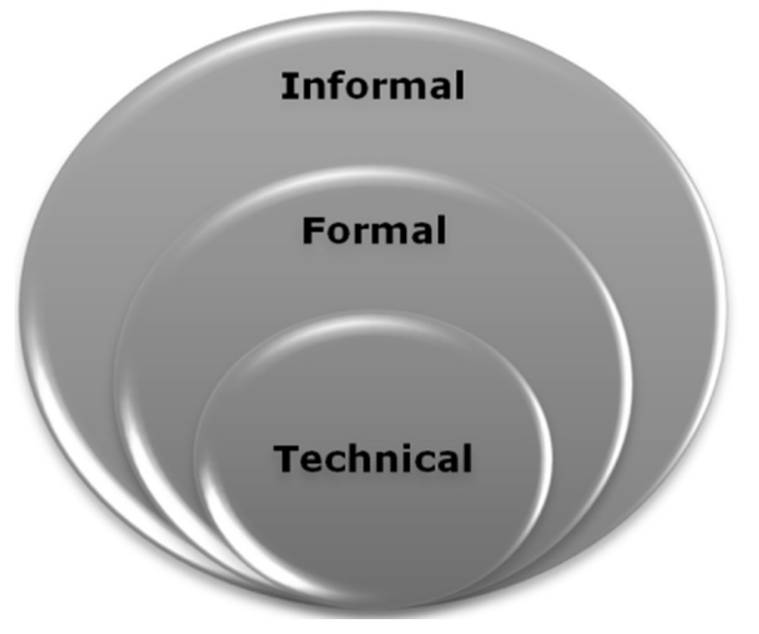

Figure 22.1. The Semiotic Onion

Organizational Semiotics [Liu 2000], [Liu and Li 2014] considers an organization and its information system as a social system in which human behaviors are organized according to a system of norms. For Stamper et al. (2000), these norms govern how the members of a community think, behave, make judgments, and perceive the world, and are directly influenced by their social context. Organizational Semiotics explores the use of signs and their effects on social practices, providing a set of methods (e.g., Problem Articulation Method, Norm Analysis Method) and artifacts (e.g., Stakeholders Identification Diagram, Semiotic Ladder, Ontology 
Charts) to deal with information and information systems in a balanced way, considering technological issues as well as human aspects of information resources, products, and functions.

In a social responsible perspective, Baranauskas $(2009,2014)$ articulates ideas inspired by Organizational Semiotics [Liu 2000] and Participatory Design [Schuler and Namioka 1993] to propose a framework that considers a dialogue with design materials and mainly among individuals in their different roles (e.g., designer, developer, user, other stakeholders) to conduct participatory work in interactive system design. For this author, the technical aspects of system design depend on and impact the formal and informal aspects of organizations and society. A technically centered perspective (i.e., focused on the onion's core) prevents those involved in a design context from a wider sense-making of the problem being handled and the solution being proposed.

Instead, Baranauskas $(2009,2014)$ argues that any design process must be understood as a movement from the outside to the inside of the Semiotic Onion: a social process that starts in society, crossing the informal and formal layers of signs towards the construction of the technical system - see the arrows in Figure 12.2, returning from the technical layer and impacting the formal and informal layers, and society. When moving from outside to inside the onion, the movement favors the identification, articulation, and formalization of relevant aspects of the social world (e.g., stakeholders' values, culture, expectations, tensions), generating new knowledge and awareness of the social context. Therefore, when returning, moving from inside to outside the onion, the movement occurs in an informed way, reflecting an understanding of the social world, making sense to stakeholders and, potentially, promoting acceptance and adoption.

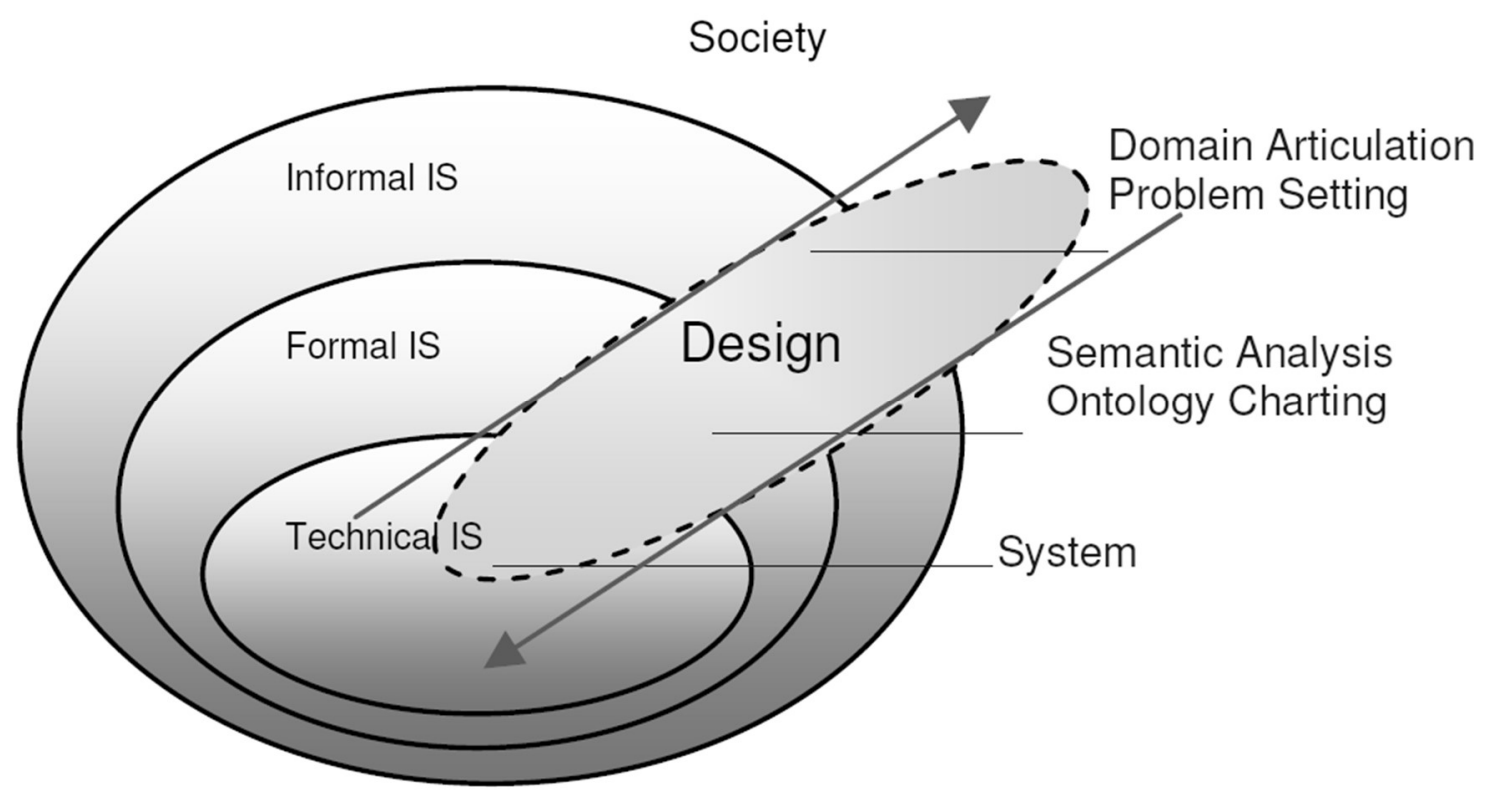

Figure 12.3. Baranauskas' (2009) Social Responsible Perspective for Design

The dashed ellipse in Figure 12.2 represents the design process of a technical information system in action: the process starts in society, i.e., it is triggered by the social world (to solve a real-world problem, to advance an existing solution, to provide a new service, etc.), it crosses the informal layer where activities are carried out to understand the problem (e.g., 
identify the stakeholders, their cultural differences, needs, interests, and expectations). Then, design progresses from the informal to formal layer where design activities support the identification and organization of requirements, the decision-making informed by the knowledge constructed during problem understanding, and the solution modeling. The process continues towards the construction of a technical system through activities that support interactive prototyping, the codification, experimentation of design alternatives, and their evaluation.

The dashed ellipse indicates that the process does not finish at the technical level, but continues crossing back to the formal and informal layers and returning to society. This means the design product will potentially trigger changes that may require updating the system model, reviewing agreements, justifying design decisions, as well as impacting established processes, formal norms and laws, and people's daily practices. Therefore, the design product also impacts the shared understanding about the problem and solution, its importance to the different stakeholders, the way activities are performed, valued or needed, and so on. The design process progresses iteratively and incrementally as much as necessary. It starts before the problem is understood and a solution is proposed, and lasts while the technical system is triggering impact - the disposal or replacement of the designed technical system is also part of the movement. Once a technical system is designed and delivered, it changes society irreversibly. Therefore, systems must be designed from a systemic and socially aware perspective, not as a mere technical component.

\subsection{The Grand Challenge}

The "Systemic and Socially Aware Perspective for Information Systems" Grand Challenge urges us to move from a technical centered perspective of information systems to one that recognizes and considers a technical solution as being part of a more complex social system in which people live, interact, develop their cultures and values, and organize their societies. Such a perspective must be informed and enriched by ethical goals, such as favoring the participative and universal access of people to knowledge [Medeiros 2008], promoting quality of life [Bannon 2011], reinforcing democracy and social participation, etc.

As we discussed previously, a technical centered perspective to IS narrows our understanding of the social context in which it is/will be delivered and operate, making it difficult to anticipate the impact and consequences of its introduction as well as obtain results that make sense to the different stakeholders directly or indirectly involved in the problem domain. It easily results in systems that violate legal norms and ethical issues - such as the case of Volkswagen ${ }^{\circ}$, that behaved quite differently from the expected or desired when the system was designed such as the case of Microsoft's Tay, and that generated difficulties for people, changing their tasks and environments in a way that did not make sense to them, triggering negative psychological, economic and social effects - as the case of the eSocial platform. Therefore, when we talk about a "systemic perspective", we talk about considering the technical system as part of the formal system, which is part of an informal system built by people in their social world.

However, a systemic perspective is not enough, as it can be "cold" disregarding its social responsibility and insensitive to the real needs of a society and its ethical concerns. When talking 
about Ambient Assisted Living, Bannon (2011) mentions how often designers and researchers develop products and carry out research hoping they will support elderly people to have a better quality of life at home instead of in an institution. However, looking more carefully, their research outcomes end up providing fulltime remote monitoring of these people instead of adding to their dignity or empowering them to remain autonomous, managing their privacy, keeping in touch with their friends. This is what usually happens when we think of technology before people, and when the concern with the key people (e.g., the elderly), their real needs, concerns and values are not actually primary, but secondary.

The design of educational technologies, especially for disabled students, usually suffers from the same "good will but narrow view" problem: designers, researchers and teachers are often interested in promoting students learning, developing their abilities, capacitating them to use technology, etc. However, although these studies usually strive for user-centered design and defend the idea of universal access and social inclusion, on looking more closely, their outcomes end up automating activities and procedures already conducted in the classroom. They expect students to achieve a "normal performance", and evaluate students based on pre-defined parameters instead of it naturally making sense to them, adding to their quality of life and promoting their welfare. Such a technical centered perspective very often blinds us to the understanding and to the design of new solutions and strategies that consider students and their particularities, so that students can develop the abilities necessary for their context of life, promoting their own progress.

As Lee (1989) argued, "neither a computer nor the teaching of computer science has any value or meaning outside of its impact on people". We think that the IS community in Brazil has a role to play and a responsibility to face: designing systems that improve the lives of people in their environments, in a way that makes sense to them and not trigger adverse effects on individuals and their communities. This naturally includes the concern for universal and participative access, designing systems that can be accessed by everyone, to the greatest possible extent, without discriminating [Baranauskas and Souza 2006]. Therefore, when we talk about a "socially aware perspective", we talk about a socially responsible, participatory and universal design of IS, both as process and product. If we want the Information Systems community to contribute to the scientific, economic and cultural development of Brazil, then we must adopt a socially aware perspective to our research, development and teaching practices.

The Brazilian context is intrinsically complicated: the country has continental dimensions, a population of more than 200 million people, a diversified culture, and is traditionally marked by social inequality. Although Brazil experienced rapid and profound transformation in the last decade, its limitations and inabilities in critical areas have become more evident (e.g. education, science and technology, healthcare, infrastructure and security, etc.). Scientific and technological development is a crucial factor in overcoming these limitations, but the benefits of development are only achieved and felt dimensions if it occurs in a socially responsible way.

Recognizing the complex and challenging context of Brazil, the Information Systems community has an important role and the unique responsibility to direct efforts towards producing studies and results that help to overcome the challenges of designing better solutions 
for our society. The Grand Challenge presented in this chapter requires a change in posture and organized work from the Information Systems community with implications and requiring effort in several dimensions, such as:

- Conceptual: there is a need for theories formalizing systemic approaches to the design of information systems, especially considering applications for all in the mobile and web domains. The explicit involvement and consideration of ethical issues require explicit articulation of different areas and fields, and must be part of such approaches. The background presented in this chapter can serve as a starting point.

- Methodological: there is a need for new process models for IS development that see IS under a systemic and socially-aware lens. New artifacts, methods and tools in line with that lens are needed to support such processes. New practices are also mandatory as social participation and engagement are key for a systemic perspective. Participatory [Schuler and Namioka 1993] and Universal Design [Preiser and Ostroff 2001] can serve as a basis to rethink current practices and inform new ones.

- Educational: the conceptual and methodological dimensions require us to rethink our IS curricula, especially for computer science, engineering and related fields, to enable our professionals not only to have a wider understanding of the products of technology they are building - sensitive to the different needs, competencies, and social values of systems - but also to an ethical understanding of the problems they are trying to solve, the methods they are using, the practices they are planning and conducting, the impacts they are triggering, and the social responsibility they have.

- Cultural: the previous dimensions demand a shift in our position while researchers, scientists, teachers, and practitioners. It demands us seeing the people prior to the problem to be solved, the social impact prior to the technology to be designed, and the actual needs of stakeholders ahead of automating tasks. Such a shift must be reflected in our research projects, methods, curricula, academic forums, debates, etc. The challenge presented in this chapter requires the IS community to embrace multidisciplinarity and actually to conduct multidisciplinary work - with other fields of Computer Science (e.g., Human Computer Interaction, Software Engineering, Computing Architecture) and other areas of knowledge (e.g., Sociology, Psychology, Linguistics). It is necessary for the community to rethink its own practices, theories and methods, which clearly have contributed to the field, but that seems not to account for the complexity of a scenario where IS is not only used, but is already part of our lives.

\subsection{Progress and Evaluation}

Concrete examples that could benefit from the results in this challenge encompass any kind of technical information systems, especially those dealing with critical or dependable information (e.g., healthcare, education, financial, security, logistic domains), easily reaching society via better services and solutions, reducing costs, improving performance, favoring access to knowledge, and so on.

Research benefits involve all society, and the Information Systems community will profit from the advances in this challenge, with new concepts, artifacts, methods, practices and tools, as well as the socialization of practical experiences and case studies. Such benefits will naturally extend to other disciplines of Computing Science concerned with the design of interactive computer technology, such as Software Engineering, Informatics in Education, and HumanComputer Interaction. Other scientific domains related to this challenge may also benefit from its advance, such as Business strategy, Economics, Sociology, Design, Education, etc. 
As suggested in the previous section, a Grand Challenge requires investigation and advances in different dimensions that are equally challenging, such as the conceptual, methodological, educational and cultural ones. The progress in this challenge's different dimensions can be promoted via public forums in local, national and international conferences dedicated to debating new concepts, theories, methods and practices for systemic and socially aware approaches - the Grand Challenges initiative conducted by the Brazilian Information Systems community is an example itself. Short courses, workshops and online communities can support discussing challenging problems, sharing and socializing new experiences and their results, generating and evaluating new knowledge, and so on. The curricula must be revisited to reinforce ethical issues, social and participatory practices, as well as to provide practical experience for the students, developing their critical thinking instead of only specialized abilities - as Einstein defended several decades ago [Fine 1952]. Experiences in real scenarios, with multidisciplinary teams and in participatory ways, are very interesting and important contexts to develop, study, analyze, apply and evaluate new concepts and methods with the potential to produce technical results in terms of the designed solutions.

Direct and indirect indicators can evaluate the progress in these dimensions, also serving as a possible list of actions to support such progress, such as:

- Number of initiatives to promote the discussion on this challenge and the dissemination of results and advances (e.g., workshops, tutorials, keynotes, panels);

- Appearance/inclusion of the challenge and related themes as topics in the call for papers of IS conferences and journals;

- Number and type of publications (books, journal and conference papers, abstracts, posters, social media contents) citing the challenge, contributing to its advance, using it as a context, criticizing it, promoting its discussion, etc.;

- Inclusion of topics related to the challenge in the curricula of information systems disciplines and courses;

- Volume of call for research projects, in terms of number and funding, that support research in the context of the Grand Challenge;

- Number and examples of technical information systems designed from the perspective proposed by the challenge;

- Initiatives that explicitly align information system development with social practices with stakeholders;

- Consideration of the challenge, and its related topics, as explicit research lines/projects of research groups;

- Number of PhDs and Masters carrying out research to advance the challenge;

- Formation of partnerships and networks between different research groups, institutions, and public and private sectors to develop research and applied work on related subjects;

- Public surveys on the nature of research projects being conducted, the kind of approaches and methods used, the background theories adopted, and so on.

Advancing a Grand Challenge requires a coordinated effort from the entire community, with periodic reviews of goals and directions, and monitoring the progress being made. It is essential for the community to keep the challenges in mind and explicit in their calls, events, discussions, teaching practices, etc., looking for partnerships with the public and private sectors in order to obtain results that benefit society. 


\subsection{Final Remarks}

In this chapter, we presented the conception and adoption of a Systemic and Socially Aware Perspective as a Grand Research Challenge for Information Systems in Brazil. We argue that we must revisit and rethink our practices in order to remain relevant and to produce effective contributions for a society which is increasingly mediated by ICTs in a country marked by social inequalities and chronic problems.

We argued and presented different examples demonstrating that a technology cannot be detached from the social context in which it was designed and delivered. Computing Information Systems, and computing artifacts in general, are produced through intentional and rational processes influenced by the background of different stakeholders, and are delivered in environments where several different stakeholders exist, who have different interests and both cause and suffer direct or indirect effects from its introduction. Computing artifacts permeate all the aspects of people's lives, not only solving problems and automating tasks, but also connecting people, mediating complex social interactions, allowing the expression of creativity, play, learning, etc. Therefore, the concern with computing artifacts cannot be restricted to their designed functions and quality attributes, but they should include a concern for the environment in which they are inserted, the people that live in that environment, the possible uses they can serve, and effects they may trigger.

In this sense, we argue that it is not possible to design computing information systems that make sense to people and their organizations, and that reflect an understanding of - and respect for - stakeholders' social world if designers pay attention solely to technical issues. It is important to begin by understanding the informal and formal aspects of the design context as profoundly and broadly as possible. Like the movement started by Papanek (1972), it is critical to challenge the way information systems are designed, moving from a technically-centered perspective to a socially and ecologically responsible design of systems, their infrastructures, and the tools used to build them. Information Systems, as a community and a discipline, can achieve its potential to contribute to society only if stakeholders are identified, understood, and involved accordingly, and if professionals are able to pay attention to the genuine needs of these stakeholders, considering the dynamics of their social context in a responsible way.

The idea of formulating Grand Research Challenges has been adopted in several countries and in different areas of knowledge. For ICT and Computing in Brazil, the Brazilian Computer Society had a pioneer initiative in 2006 [Medeiros 2006], and the Human-Computer Interaction community conducted a similar initiative specifically for its field in 2012 [Baranauskas et al. 2015]. Both the initiatives seem to have helped in the formulation of a longterm research agenda, influencing researchers, practices, conferences, and even public policies on research funding, as happened with 2006's Grand Challenges. We hope this initiative is the first one in a series of events and publications. We believe it can contribute to significant changes and advances not only in the domain of Information Systems, but also in Computer Science and its applications, mainly with regard to producing interactive technology for the scientific, economic and social development of the country, in a participatory and universal way.

\section{Acknowledgements}

This research was partially funded by São Paulo Research Foundation - FAPESP (Grant 
\#2015/16528-0). The authors thank the organizing committee of the Grand Challenges in Information Systems initiative, and the opportunity to write this Grand Challenge chapter. The authors also thank their institutions, research groups, colleagues and students. M. Cecilia C. Baranauskas has a productivity scholarship from CNPq (Grant \#308618/2014-9).

\section{References}

Bannon, L. (2011) "Reimagining HCI: toward a more human-centered perspective". Interactions 18, 4: 50-57.

Baranauskas, M.C.C., Souza, C.S., Pereira, R. (2015) "I GranDIHC-BR - Grand Research Challenges in Human-Computer Interaction in Brazil". Brazilian Computer Society (SBC). ISBN: 978-85-7669-296-6. 61p.

Baranauskas, M.C.C. (2014) "Social awareness in HCI”. Interactions 21, 4, p.66-69.

Baranauskas, M.C.C. (2009) "Socially Aware Computing”. In Proc. of VI International Conference on Engineering and Computer Education (ICECE), p. 1-5.

Baranauskas, M.C.C. and de Souza, C.S. (2006) "Desafio 4: Acesso participativo e universal do cidadão brasileiro ao conhecimento". Computação Brasil, ano VII, (23), p.7. (In Portuguese)

Becker, J., vom Brocke, J., Heddier, M. and Seidel, S. (2015) In search of information systems (grand) challenges. Business \& Information Systems Engineering, 57(6), p.377-390.

Bødker, S. (2006) "When second wave HCI meets third wave challenges". In Proceedings of 4th Nordic conference on HCI: changing roles, p. 1-8.

Fine, B. (1952) "Einstein Stresses Critical Thinking”. The New York Times. Interview published on October 5, 1952.

Hall, E.T. “The Silent Language”. Anchoor Books. 1959.

Lee, E. (1989) "Some suggestions on a computer science undergraduate curriculum". In COMPCON Spring'89. Thirty-Fourth IEEE Computer Society International Conference: Intellectual Leverage, Digest of Papers. p. 366.

Liu, K. and Li, W. “Organisational Semiotics for Business Informatics”. Routledge. 2014.

Liu, K. "Semiotics in Information Systems Engineering”. Cambridge University Press. 2000 .

Medeiros, C.B. (2008) "Grand Research Challenges in Computer Science in Brazil". IEEE Computer, 41(6), p.59-65.

Papanek, V. "Design for the real world". London. Thames and Hudson. 1971.

Pereira, R. and Baranauskas, M.C.C. (2015) "A value-oriented and culturally informed approach to the design of interactive systems". International Journal of HumanComputer Studies 80 (August): 66-82.

Pereira, R., Baranauskas, M.C.C. and Liu, K. (2015) "The Value of Values for HCI: an informed discussion beyond philosophy". XIV Brazilian Symposium on Human Factors in Computer Systems (IHC 2015). p. 430-439.

Preiser, W.F. and Ostroff, E. "Universal design handbook". McGraw Hill Professional. 2001. 
Schuler, D. and Namioka, A. "Participatory design: principles and practices". Lawrence Erlbaum Associate. 1993.

Sellen, A., Rogers, Y., Harper, R. and Rodden, T. (2009) "Reflecting human values in the digital age". Communications of the ACM, 52, p.58-66.

Stamper, R., Liu, K., Hafkamp, M. and Ades, Y. (2000) "Understanding the Role of Signs and Norms in Organisations - a semiotic approach to information systems design". Journal of Behaviour and Information Technology 19, 1: 15-27.

Walsham, G. (2012) “Are we making a better world with ICTs? Reflections on a future agenda for the IS field”. Journal of Information Technology, 27(2), p.87-93.

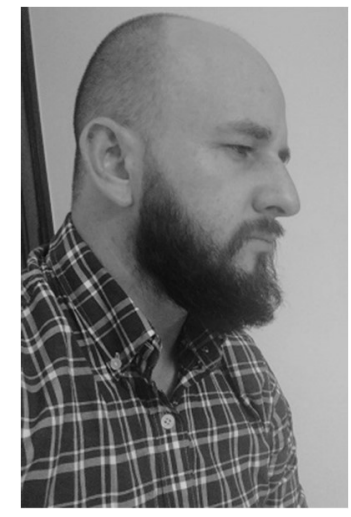

\section{Roberto Pereira}

CV: http://lattes.cnpq.br/1607596289960868

$\mathrm{PhD}$ in Computer Science, Roberto Pereira did his Post-doctorate at the University of Campinas and was a Visiting Postdoctoral Research Fellow at the University of Reading - UK. Roberto is an adjunct professor and researcher at the Department of Computer Science, Federal University of Paraná (UFPR), and an Associate Editor of the Brazilian Journal of Computers in Education (RBIE). His research is on the boundaries of HumanComputer Interaction, Software Engineering and Information Systems, also contributing to Informatics in Education and Organizational Semiotics. e-mail: rpereira@inf.ufpr.br

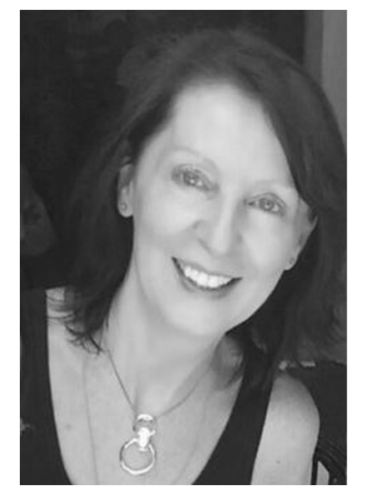

\section{Cecilia C. Baranauskas}

CV: http://lattes.cnpq.br/1750385790843118

Maria Cecilia Calani Baranauskas did post-doctorate research at the Universities of Reading and Staffordshire (UK), and the Polytechnic University of Madrid (Spain). She holds a degree in Computer Science and Mathematics, Master's degree in Computer Science, and a $\mathrm{PhD}$ in Electrical Engineering from UNICAMP. Cecilia is a Full Professor at the Institute of Computing, UNICACVMP. Her research contributes to the areas of HCI, Organizational Semiotics, and design of interactive systems in different domains (social, educational, work). Cecília received several best paper awards as well as awards for her lifetime contribution, such as the Educational Merit Diploma "Professor Darcy Ribeiro", the ACM SIGDOC Rigo Award, and the 1st Outstanding Career in HCI Award, from the Brazilian Computer Society's Special Group of HCI. e-mail: cecilia@ic.unicamp.br 